\title{
Chromosomal Aberrations and HMGA2 Expression in Paroxysmal Nocturnal Hemoglobinuria
}

\author{
Sellmann L ${ }^{1,2}$, Klein-Hitpass L ${ }^{2}$, Gesk $S^{3}$, Nückel $H^{1}$, Dührsen $\mathrm{U}^{1}$, Scholtysik $\mathrm{R}^{* \neq 2}$ and Röth $\mathrm{A}^{\# 1}$ \\ ${ }^{1}$ Department of Hematology, Faculty of Medicine, University Duisburg-Essen, Essen, Germany \\ ${ }^{2}$ Institute of Cell Biology (Cancer Research), Faculty of Medicine, University Duisburg-Essen, Essen, Germany \\ ${ }^{3}$ Institute of Human Genetics, Christian-Albrechts-University Kiel \& University Hospital Schleswig-Holstein, Kiel, Germany \\ \#These authors contributed equally
}

*Corresponding author: Scholtysik R, Institute of Cell Biology (Cancer Research), University Hospital Essen, Virchowstraße 173, D-45122 Essen, Germany, Fax: +49 201723 3386, Tel: +49 201723 3389, E-mail: rene. scholtysik@uni-due.de

Citation: Sellmann L, Klein-Hitpass L, Gesk S, Nückel H, Dührsen U, et al. (2015) Chromosomal Aberrations and HMGA2 Expression in Paroxysmal Nocturnal Hemoglobinuria. J Hematol Blood Disord 2(1): 101. doi: 10.15744/2455-7641.2.101

Received Date: July 16, 2015 Accepted Date: August 27, 2015 Published Date: September 01, 2015

\begin{abstract}
Somatic, inactivating PIGA mutations in haematopoietic stem cells, followed by an unknown autoimmune selection process in favor of the mutated clone, are thought to be important events in the pathogenesis of paroxysmal nocturnal hemoglobinuria (PNH). Recently, a second clonal event involving the HMGA2 gene was reported in some PNH cases, and over expression of this gene was postulated to promote proliferation of the mutated clone. The present study investigated a total of $37 \mathrm{PNH}$-patients with two goals: First, single nucleotide polymorphism chip analysis was performed to detect novel recurrent copy number changes or allelic imbalances. A single deletion affecting the PIGA locus was found, but no other relevant or recurrent abnormalities were detected. Thus, although PIGA mutations are established as pathogenetic drivers of $\mathrm{PNH}$, larger deletions of the locus are rare. Second, fluorescence in situ hybridization was conducted for the region containing the HMGA2 gene in addition to expression analysis, to study the recurrence of changes at this locus. This revealed no aberration of the chromosomal region containing the HMGA2 gene in the cohort, and HMGA2 expression was surprisingly only detectable in a single case.

Keywords: PNH; SNP chips; Chromosomal aberrations; HMGA2

Abbreviations: BAC: Bacterial Artificial Chromosome; FISH: Fluorescence in-situ Hybridization; MNC: Mononuclear Cells; PNH: Paroxysmal Nocturnal Hemoglobinuria; SNP: Single Nucleotide Polymorphism; UPD: Uniparental Disomy
\end{abstract}

\section{Introduction}

Paroxysmal nocturnal hemoglobinuria $(\mathrm{PNH})$ is an acquired clonal hematologic disorder with typical clinical manifestations, including intravascular hemolysis, venous thrombosis, and defective hematopoiesis [1]. Biochemically, PNH is a consequence of nonmalignant clonal expansion of hematopoietic stem cells with somatic mutation of PIGA [2,3], and mutant, inactive PIGA explains the deficiency of glycosyl phosphatidylinositol-anchored proteins [4]. Furthermore, preexisting bone marrow failure to various degrees seems to be required for the development of PNH [5,6]. PNH clones are present in a majority of patients with aplastic anemia, which is consistent with the strong immune component in the pathogenesis of this disease [7-9]. Furthermore, inhibition of the complement cascade is efficient in treating PNH-related symptoms [10]. Interestingly, the presence of a low number of PNH cells, even in healthy individuals, confirms the hypothesis that defective stem cells actually predate the stem cell failure and depletion [11]. Unlike dysplastic clones in myelodysplastic syndrome (MDS), which harbour intrinsic defects that predispose the cells to clonal evolution, external factors, such as pressure exerted by the immune system, have for a long time been speculated to be the main causes for clonal escape in $\mathrm{PNH}$ [5].

Inoue, et al. were the first to report a possible second clonal event. They described two patients with PNH with an acquired rearrangement of chromosome 12 affecting the HMGA2 gene, which encodes a chromatin remodeling protein, in the PIGA mutant cells [12]. HMGA2 is located in an area on chromosome 12q13 frequently involved in translocations and amplifications in benign, mesenchymal tumors. Emerging evidence also indicates an important role for HMGA2 overexpression in tumorigenesis, including poorly differentiated solid tumors and some cases of leukemia [13-16]. The two PNH patients from the Inoue, et al. study had ectopic expression of HMGA2 in the bone marrow. Based on this finding, HMGA2 was considered to be a candidate driver gene, as ectopic expression induced cellular proliferation [12]. Another study identified additional PNH cases exhibiting elevated HMGA2 expression [17]. However, the importance of HMGA2 expression in PNH remains controversial [18]. Shen, et al. have recently sequenced the exome of affected cells in $12 \mathrm{PNH}$ patients, and found a complex pattern of clonal evolution denoted by shared and distinct mutations in subclones, but not a single recurrent mutation besides PIGA [19]. 
Detection of further chromosomal aberrations in PNH clones has been hampered by the low mitotic index of cells, purity of samples, and coexistence of subclones [20]. Single nucleotide polymorphism (SNP) chips have the advantage of detecting aberrations in non-dividing cells and reveal genomic imbalances at a very high resolution. Furthermore, they are able to detect copy number-neutral loss of heterozygosity events, which are called (partial) uniparental disomies (UPD) [21]. Their resolution is also much greater than that of routine metaphase cytogenetics. SNP-based cytogenetic analysis of unbalanced genomic defects can be applied to study a variety of malignancies and germline defects [5,22,23].

Hence, the aim of the present study was to identify novel chromosomal aberrations detectable by SNP chips, to search for chromosomal aberrations involving the HMGA2 gene by fluorescence in situ hybridization (FISH) and to investigate levels of HMGA2 expression in PNH patients.

\section{Patients and Methods}

\section{Patients}

Thirty-seven patients with PNH from the Department of Hematology of the University of Duisburg-Essen who were diagnosed according to the World Health Organization (WHO) criteria were selected for this retrospective, single-centre analysis. Comprehensive clinical information, including treatment history, was available for all patients.

\section{Samples}

Peripheral venous blood samples in EDTA were obtained according to institutional guidelines. Peripheral blood mononuclear cells (MNC) were separated by density gradient centrifugation using Ficoll-Hypaque (Pharmacia, Freiburg, Germany) and cryopreserved until further analysis. Granulocytes were isolated from fresh blood by using Polymorphprep (Axis-Shield, Oslo, Norway). Immunophenotypic analysis by flow cytometry was employed to document the percentage of abnormal GPI-deficient granulocytes as previously described [24]. RNA and DNA were extracted by using the respective QIAmp blood kits (Qiagen, Hilden, Germany) according to the manufacturer's instructions. To retain RNA quality, RNA extraction was performed on ice.

\section{FISH studies}

FISH was performed on peripheral blood cells from 30 patients. For the detection of aberrations involving the HMGA2 gene at chromosome 12q14.3, bacterial artificial chromosome (BAC) clones were selected by using bioinformatic resources available at the University of California at Santa Cruz website (http://genome.ucsc.edu/cgi-bin/hgGateway) as follows: RP11-125F18 (centromeric to the HMGA2 gene locus, labelled in spectrum orange); RP11-462A13 (spanning the HMGA2 gene locus, labelled in spectrum green); and RP11-945G8 (telomeric to HMGA2, labelled in spectrum green). BAC clones were prepared, BAC DNA was labelled, and the hybridization, washing, and evaluation steps were performed according to standard protocols, as previously described [25]. For the determination of the diagnostic thresholds of the new HMGA2 probe set, 200 nuclei from blood samples of five healthy persons were evaluated to serve as negative controls. The cut-off level for gains or translocations involving the HMGA2 gene locus was calculated as the mean of false-positive nuclei in controls plus three standard deviations and was set as $6 \%$.

\section{SNP chip analysis}

For SNP array studies, genomic DNA was extracted from granulocytes isolated from 15 blood samples of PNH patients (cell number: $3.2 \pm 1.6 \times 10^{7}$ ), 7 buccal swabs from PNH patients (as imperfect germline DNA control; contamination by blood cell DNA cannot be excluded), and granulocytes from the peripheral blood samples of 6 normal donors (as normal reference) with the QIAamp blood kit (Qiagen, Hilden, Germany), according to the manufacturer's instructions. Array experiments were performed according to the standard protocol for Affymetrix GeneChip SNP 6.0 arrays (Affymetrix, Santa Clara, CA, USA). Briefly, a 500-ng sample of DNA was digested with StyI and NspI, ligated to adaptors, amplified by PCR, fragmented with DNAse I, and biotinlabelled. The labelled samples were hybridized to the Affymetrix GeneChip SNP 6.0 arrays, followed by washing, staining, and scanning.

\section{Copy number and UPD analysis}

The acquired signal data were loaded into the Affymetrix Genotyping Console Software, version 4 (Affymetrix). Genotyping and copy number analysis were performed with the manufacturer-supplied model and reference files for SNP 6.0 arrays, employing the human genome version 18. Parameters for the algorithms were left at their default values. The built-in Genome Browser was used for visualization of the detected aberrant regions.

\section{Real-time RT-PCR for HMGA2}

Total RNA was extracted from 25 samples from PNH patients, purified with the RNeasy Midi Kit (Qiagen), and quantified spectrophotometrically as previously described [26]. In eight patients where the purification of granulocytes was not successful, MNC were used instead (see Table 1). As a positive control, RNA was extracted from CD $34^{+}$cells from one patient without PNH. RNA from granulocytes of five healthy donors was used as a normal reference. The RNA isolation procedure included an oncolumn digestion step of residual genomic DNA using DNase I, as recommended by the manufacturer. One microgram of total 
RNA was used for cDNA synthesis with oligo-dT primers (Roche, Mannheim, Germany) and Superscript II reverse transcriptase (Invitrogen, Karlsruhe, Germany) [26]. Quantification of HMGA2 was carried out by using a forward primer located in exon 2 (5'-GTCCCTCTAAAGCAGCTCAAAA-3') and a reverse primer located in exon 5 (5'-CTCCCTTCAAAAGATCCAACTG-3'). All primers were manufactured by MWG Biotech (Ebersberg, Germany). PCR and primers for beta-actin were applied as previously described [26]. The PCR reaction mix was prepared with the Quantitect SYBR Green kit (Qiagen), according to the manufacturer's instructions. PCR was performed in duplicate on the ABI 7500 Real Time PCR System (Applied Biosystems, Foster City, USA). An annealing temperature of $57^{\circ} \mathrm{C}$ for $33 \mathrm{~s}$ and an extension step at $72{ }^{\circ} \mathrm{C}$ for $33 \mathrm{~s}$ were used. The relative expression of $H M G A 2$ in relation to beta-actin was quantified by using the formula $H M G A 2 /$ beta-actin $=2^{\text {-deltaCt }}$.

\begin{tabular}{|c|c|c|c|c|c|c|c|c|c|}
\hline No. & Sex & $\begin{array}{c}\text { Age at } \\
\text { diagnosis } \\
\text { (years) }\end{array}$ & $\begin{array}{l}\text { Aplastic } \\
\text { anemia }\end{array}$ & $\begin{array}{c}\text { Thrombembolic } \\
\text { history }\end{array}$ & $\begin{array}{c}\text { Eculizumab } \\
\text { therapy }\end{array}$ & Purity & FISH & $\begin{array}{c}\text { HMGA2 delta } \\
\text { CT }\end{array}$ & SNP-chip \\
\hline 1 & M & 25 & no & & yes & 88 & inconspicuous & undetermined & $46 \mathrm{XY}$ \\
\hline 2 & M & 24 & no & & yes & 80 & inconspicuous & $23.30^{\mathrm{a}}$ & nd \\
\hline 3 & M & 38 & no & DVT, MI & yes & $\mathrm{MNC}$ & inconspicuous & 19.18 & nd \\
\hline 4 & $\mathrm{~F}$ & 17 & no & & yes & 98 & inconspicuous & undetermined & nd \\
\hline 5 & M & 19 & yes & & yes & 87 & inconspicuous & undetermined & $46 \mathrm{XY}$ \\
\hline 6 & M & 42 & no & & yes & nd & inconspicuous & undetermined & nd \\
\hline 7 & $\mathrm{~F}$ & 54 & no & DVT & yes & 97 & inconspicuous & undetermined & nd \\
\hline 8 & M & 15 & no & & yes & 99 & nd & undetermined & 46XY,-Xp22.2 \\
\hline 9 & $\mathrm{~F}$ & 15 & no & & yes & 97 & nd & undetermined & nd \\
\hline 10 & M & 19 & yes & & yes & 85 & inconspicuous & undetermined & $46 \mathrm{XY}$ \\
\hline 11 & $\mathrm{M}$ & 27 & no & & yes & 91 & inconspicuous & undetermined & $46 \mathrm{XY}$ \\
\hline 12 & M & 77 & no & DVT & yes & MNC & inconspicuous & $20.97^{a}$ & nd \\
\hline 13 & $\mathrm{~F}$ & 42 & yes & & yes & 88 & inconspicuous & $21.18^{a}$ & $46 \mathrm{XX}$ \\
\hline 14 & $\mathrm{~F}$ & 26 & no & $\mathrm{PE}$ & yes & MNC & inconspicuous & undetermined & nd \\
\hline 15 & M & 11 & yes & Thrombotic tumor ${ }^{\mathrm{b}}$ & yes & MNC & inconspicuous & nd & nd \\
\hline 16 & $\mathrm{~F}$ & 18 & no & DVT & yes & 67 & inconspicuous & undetermined & $46 \mathrm{XX}$ \\
\hline 17 & M & 80 & yes & & yes & 87 & inconspicuous & undetermined & $46 \mathrm{XY}$ \\
\hline 18 & $\mathrm{~F}$ & 59 & no & & yes & MNC & inconspicuous & undetermined & nd \\
\hline 19 & $\mathrm{~F}$ & 25 & no & DVT, port thrombosis & yes & 92 & inconspicuous & undetermined & $46 \mathrm{XX}$ \\
\hline 20 & $\mathrm{~F}$ & 54 & no & & yes & $\mathrm{MNC}$ & inconspicuous & undetermined & nd \\
\hline 21 & $\mathrm{~F}$ & 31 & yes & & no & $\mathrm{MNC}$ & inconspicuous & undetermined & nd \\
\hline 22 & M & 38 & no & & yes & MNC & inconspicuous & nd & nd \\
\hline 23 & M & 32 & no & & no & MNC & inconspicuous & nd & nd \\
\hline 24 & M & 21 & no & & no & 74 & inconspicuous & nd & nd \\
\hline 25 & M & 37 & no & & no & $\mathrm{MNC}$ & inconspicuous & nd & nd \\
\hline 26 & $\mathrm{~F}$ & 18 & no & & no & MNC & nd & undetermined & nd \\
\hline 27 & M & 40 & no & & no & MNC & inconspicuous & $27.40^{\mathrm{a}}$ & nd \\
\hline 28 & M & 41 & no & DVT & yes & 79 & inconspicuous & undetermined & $46 \mathrm{XY}$ \\
\hline 29 & $\mathrm{~F}$ & 23 & no & & yes & 83 & inconspicuous & undetermined & nd \\
\hline 30 & $\mathrm{~F}$ & 20 & no & & yes & 83 & inconspicuous & undetermined & $46 \mathrm{XX}$ \\
\hline 31 & M & 18 & no & & no & 71 & nd & nd & $46 \mathrm{XY}$ \\
\hline 32 & M & 15 & no & DVT & no & 85 & nd & nd & $46 \mathrm{XY}$ \\
\hline 33 & $\mathrm{~F}$ & 43 & no & DVT & yes & 92 & nd & nd & $46 \mathrm{XX}$ \\
\hline 34 & M & 34 & yes & MI & yes & 59 & nd & nd & $46 \mathrm{XY}$ \\
\hline 35 & M & 21 & no & & yes & MNC & inconspicuous & nd & nd \\
\hline 36 & M & 25 & yes & & no & MNC & inconspicuous & nd & nd \\
\hline 37 & $\mathrm{~F}$ & 32 & yes & & yes & 83 & inconspicuous & nd & nd \\
\hline
\end{tabular}

Table 1: Clinical data and Results

Purity PNH clone size in extracted granulocytes, MNC mononuclear cells were investigated for HMGA2 PCR as not sufficient granulocytes were extractable, M male, F female, DVT deep vein thrombosis, MI myocardial ischemia, PE pulmonary embolism, nd not done, HMGA2 delta CT HMGA2/beta-actin=2-detact

${ }^{\text {a }}$ very low amounts of HMGA2 RNA and detected only in one of the duplicates

${ }^{\mathrm{b}}$ Thrombotic tumor right heart 


\section{Results}

The clinical data of $37 \mathrm{PNH}$ patients (22 male; 59\%) are summarized in Table 1. Patients had a median age of 26 years at the time of primary diagnosis. During the clinical course, 28 patients (76\%) received eculizumab therapy and 16 patients (43\%) were enrolled in clinical studies. Treatment was initiated, if indicated, at a median age of 37 years. Eleven patients (30\%) suffered from thromboembolic complications and 9 patients (24\%) had concomitant aplastic anemia.

To unravel novel and possibly recurrent chromosomal aberrations, SNP chip analysis was performed when enough DNA was obtained, resulting in 15 patients analyzed. The mean purity of granulocytes from which DNA was extracted after Ficoll Hypaque centrifugation was $88 \% \pm 7 \%$. The calculated PNH mean clone size was $83 \% \pm 9 \%$, which was determined by flow cytometry with fluorescent aerolysin. There is no accepted percentage of cell purity required to detect an abnormality by SNP chip analysis because the detection threshold depends on the size and magnitude of the aberrations. However, as described by Tiu, et al. and in accordance with our experience, at least $70 \%$ of cells should carry a monoallelic lesion to be reliably detected [5], which was a threshold in PNH clone purity surpassed in all PNH samples analyzed in this study.

In 7 of the 15 PNH patients, buccal swabs yielded sufficient DNA for simultaneous SNP chip analysis of the germline. As another reference for comparison, DNA from 6 healthy volunteers was extracted after granulocyte enrichment with the same method as was used for the PNH patients. One male PNH patient showed a deletion of $182 \mathrm{~kb}$ within the region Xp22.2 (Table 1; patient No. 8). Although the coding part of the PIGA gene itself was not deleted according to the copy number analysis, this aberration deleted material located upstream of the gene, which could remove portions of the promoter or other regulatory elements (Figure 1). However, no further relevant chromosomal aberrations, such as deletions, insertions, or UPDs, were detected in this case or in the other investigated PNH patients.

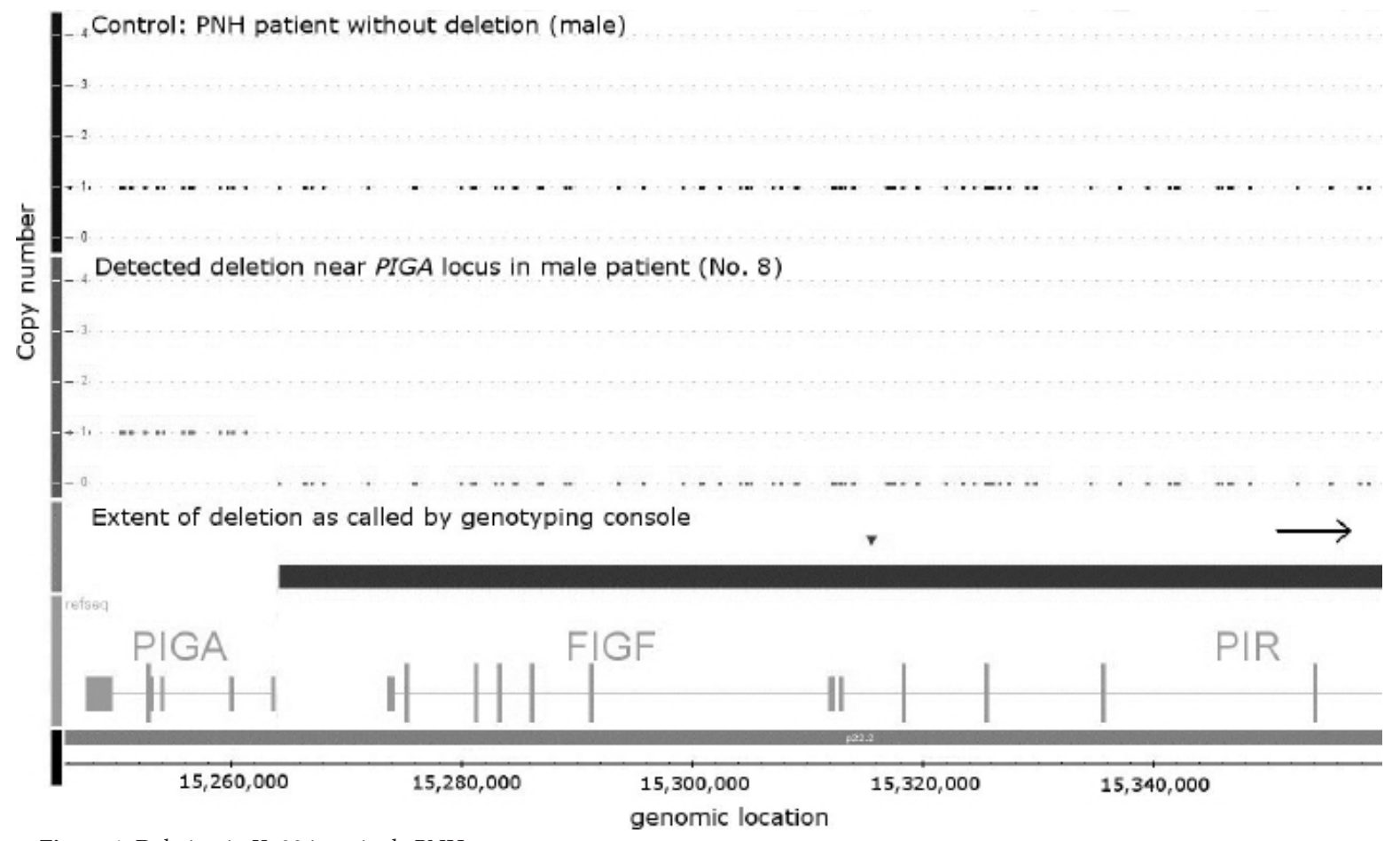

Figure 1: Deletion in Xp22 in a single PNH case

Fifteen PNH cases were evaluated by SNP chip analysis. PNH patient No. 8 exhibited a deletion of $182 \mathrm{~kb}$ in the region Xp22.2 (dotted line: copy number values for SNP probes in this region). One end of the deletion lies very close to the first exon of the PIGA gene, which could possibly remove regulatory or promoter sequences. For comparison, another SNP profile without gain or loss is shown above. The deletion is longer than the region displayed here

Cells from the peripheral blood of 30 patients were investigated by FISH for imbalances and structural aberrations involving the HMGA2 gene locus in chromosomal region 12q14.3. All 30 samples showed inconspicuous FISH results for the HMGA2 gene locus (Table 1). The HMGA2 gene was originally postulated to be a candidate gene for PNH pathogenesis because it was found to be overexpressed in a PNH patient [17]. Interestingly, after quantitative RT-PCR of granulocytes or, if sufficient RNA could not be extracted, of MNC, significant expression of HMGA2 in investigated cells was only detected in 1 out of 21 (5\%) patients (Table 1; Patient No. 3). In 4 additional PNH patients (19\%), very low amounts of HMGA2 RNA were detected in one of the duplicates. The other 16 samples from PNH patients had no HMGA2 expression at all, which was in concordance with control peripheral blood cells from five healthy donors. Moreover, the positive control (CD34-positive cells from a patient without PNH) showed strong expression of the gene, at a CT-value of 9.55 . 


\section{Discussion}

In addition to the known PIGA mutation that has been found in hematopoietic stem cells, followed by a speculative mechanism of immunological selection in favor of the PNH clone [6], a second clonal event was recently postulated in the pathogenesis of PNH. Two patients with PNH had a concurrent rearrangement of chromosome 12 affecting the HMGA2 gene, which led to elevated expression of HMGA2 compared to healthy volunteers [12]. However, the search for further chromosomal aberrations has been hampered by the low mitotic potency of cells harboring the PNH clone. Genome-wide SNP array-based methods could help to identify novel chromosomal aberrations that exclude reciprocal events. Indeed, we detected a deletion in Xp22.2 in one male patient out of $15 \mathrm{PNH}$ patients in the present study. The affected region had a size of $182 \mathrm{~kb}$, and begins upstream of the PIGA locus. It is tempting to speculate that this aberration is the cause for the loss of PIGA expression in this patient, although no firm conclusion can be reached with the data presented here. The other 14 patients analysed by SNP-Chip had no discernable chromosomal aberration, which is in contrast with another study of PNH patients that reported trisomy 6 and monosomy 7 in affected cells [27].

Total deletions of the PIGA gene have been rarely described, and most reported deletions were small [28-30], with only a single publication finding a large deletion of the entire locus [31]. Searching the Mitelman database for the occurrence of PIGA aberrations or alterations in Xp22 revealed genetic changes in one case of chronic myeloid leukemia (CML), one case of MDS, one case of chondroid hamartoma of the lung, one case each of adenocarcinoma of the prostate and ovary, three cases of acute lymphoblastic leukemia (ALL), and two cases of acute myeloblastic leukemia (AML), but no cases of PNH. All aberrations identified were translocations, with the exception of one ALL case, which was ins (X;12) (p22;q13q14). Interestingly, this case involved the HMGA2 gene [32]. Therefore, somatic mutations of the PIGA gene remain the main mechanism of PIGA disruption. These mutations are located throughout the entire coding region, and most are frameshift mutations producing at most a nonfunctional PIGA protein $[19,29]$.

Inoue, et al. described two patients with rearrangements of chromosome 12 that affected the HMGA2 gene. Searching the Mitelman database for HMGA2 revealed 153 cases with clonal aberrations encompassing HMGA2. These cases included mostly benign illnesses, such as hamartoma, lipoma, and adenoma. However, myeloid neoplasms were also described [5,16,32]. HMGA2 aberrations could also be clonal events in PNH. In this study, to estimate the frequency of $H M G A 2$ rearrangements, $30 \mathrm{PNH}$ patients were evaluated with a specifically designed FISH probe for screening. However, no translocation was detected, demonstrating that this event is not common in PNH. In patients with concomitant PNH and MDS, other studies have found trisomy 8 in the fraction of GPI-deficient cells, suggesting that the PIGA mutation affected the stem cell first, and chromosomal aberrations evolved within a subclone thereafter $[5,33,34]$. Recently, it was shown that a stochastic model of clonal stem cell evolution could capture some of the features of PNH [35]. However, the exact mechanism of clonal dominance remains unclear in most PNH patients, and secondary clonal events seem to be rare, in line with recent findings by whole exome sequencing of PNH affected cells [19].

Overexpression of HMGA2 was first reported in patients with myeloproliferative neoplasia [13]. All of these patients had translocations or inversions involving chromosomal bands 12q13-15 that resulted in the overexpression of HMGA2. Furthermore, truncated HMGA2 transcripts containing only exons 1-3 have been reported to have oncogenic properties [36]. Murakami, et al. found significantly higher HMGA2 expression in the peripheral blood of PNH patients than in healthy persons. However, there was no significant difference in the relative expression of HMGA2 in the bone marrow [17]. Additionally, analyzing the genomic sequence of three patients, including one who had the highest HMGA2 expression, revealed no mutation in the 3' untranslated region [17]. Two studies in transgenic mice overexpressing HMGA2 lead the respective authors to speculate that overexpression of HMGA2 leads to proliferative hematopoiesis with clonal expansion at the stem cell and progenitor levels and may account for the clonal expansion in PNH [17,37]. However, the clinical course of the mice was very benign, and the proportion of PNH cells was remarkably stable. Therefore, it was suggested that HMGA2 overexpression itself may not be sufficient to induce clonal expansion of haematopoietic progenitor cells $[18,38]$. The present study found relevant HMGA2 expression in 1 of $21 \mathrm{PNH}$ patients (5\%). Another $4 \mathrm{PNH}$ patients showed very low HMGA2 expression. These data suggest that HMGA2 expression may at most be important in a small fraction of PNH patients, although the expression of shorter or truncated transcripts with unclear relevance cannot be excluded with the primers used in this study.

Clonal expansion has been proposed to be a result of genetic, epigenetic, immunologic, or stochastic events that, together with mutant PIGA, provide a proliferative advantage to the mutant PNH cells [39]. Recently, an autoreactive, CD1d-restricted, GPIspecific T-cell population was found to be expanded in PNH patients and was postulated as being responsible for bone marrow failure in $\mathrm{PNH}$, indicating the importance of an autoimmune component in $\mathrm{PNH}$ [40].

Taken together, the present work shows that chromosomal aberrations could only be detected in single PNH cases by SNP chip analysis. We identified one PNH patient with the largest deletion adjacent to the PIGA gene region reported to date. Relevant novel or recurrent abnormalities were not detectable by SNP chip analysis. A second clonal event affecting the HMGA2 gene was also not detectable in the present study and seems to be uncommon in PNH. It cannot be excluded that HMGA2 expression plays a pathophysiological role in those PNH patients with overexpression of this gene, but this work suggests that this is not a regular feature in $\mathrm{PNH}$. 


\section{Acknowledgments}

This work was supported from the IFORES program of the University Duisburg Essen (L.S.) and the German Ministry of Education and Research (BMBF) through the HaematoSys project (grant 0315452G). We thank Prof. Reiner Siebert (Institute of Human Genetics, Christian-Albrechts-University Kiel, Germany) and Prof. Ralf Küppers, (Institute for Cell Biology, University DuisburgEssen, Germany) for support of the project and critical reading of the manuscript.

\section{Authors' Contributions}

A.R. and L.S. designed the research; L.S., S.G., L.K.H., H.N., R.S., and A.R. performed the experiments and collected the data; L.S., L.K.H., S.G., R.S., H.N., U.D. and A.R. analyzed and interpreted the data; L.S., R.S. and A.R. wrote the paper.

\section{Compliance with Ethical Standards}

The authors declare no conflict of interest. The study was approved by the Ethics Committee of the Faculty of Medicine at the University of Duisburg-Essen and is in accordance with the Helsinki Declaration of 1975 and its later amendments. All patients provided written informed consent.

\section{References}

1. Ware RE, Hall SE, Rosse WF (1991) Paroxysmal nocturnal hemoglobinuria with onset in childhood and adolescence. N Engl J Med 325: 991-6.

2. Takeda J, Miyata T, Kawagoe K, Iida Y, Endo Y, et al. (1993) Deficiency of the GPI anchor caused by a somatic mutation of the PIG-A gene in paroxysmal nocturnal hemoglobinuria. Cell 73: 703-11.

3. Ware RE, Rosse WF, Howard TA (1994) Mutations within the Piga gene in patients with paroxysmal nocturnal hemoglobinuria. Blood 83: 2418-22.

4. Parker C, Omine M, Richards S, Nishimura J, Bessler M, et al. (2005) Diagnosis and management of paroxysmal nocturnal hemoglobinuria. Blood 106: $3699-709$.

5. Tiu R, Gondek L, O’Keefe C, Maciejewski JP (2007) Clonality of the stem cell compartment during evolution of myelodysplastic syndromes and other bone marrow failure syndromes. Leukemia 21: 1648-57.

6. Luzzatto L, Bessler M, Rotoli B (1997) Somatic mutations in paroxysmal nocturnal hemoglobinuria: a blessing in disguise? Cell 88: 1-4.

7. Young NS, Calado RT, Scheinberg P (2006) Current concepts in the pathophysiology and treatment of aplastic anemia. Blood 108: 2509-19.

8. Wlodarski MW, Gondek LP, Nearman ZP, Plasilova M, Kalaycio M, et al. (2006) Molecular strategies for detection and quantitation of clonal cytotoxic T-cell responses in aplastic anemia and myelodysplastic syndrome. Blood 108: 2632-41.

9. Chen G, Zeng W, Maciejewski JP, Kcyvanfar K, Billings EM, et al. (2005) Differential gene expression in hematopoietic progenitors from paroxysmal nocturnal hemoglobinuria patients reveals an apoptosis/immune response in 'normal' phenotype cells. Leukemia 19: 862-8.

10. Röth A, Hock C, Konik A, Christoph S, Dührsen U (2011) Chronic treatment of paroxysmal nocturnal hemoglobinuria patients with eculizumab: safety, efficacy, and unexpected laboratory phenomena. Int J Hematol 93: 704-14.

11. Araten DJ, Nafa K, Pakdeesuwan K, Luzzatto L (1999) Clonal populations of hematopoietic cells with paroxysmal nocturnal hemoglobinuria genotype and phenotype are present in normal individuals. Proc Natl Acad Sci U S A 96: 5209-14.

12. Inoue N, Izui-Sarumaru T, Murakami Y, Endo Y, Nishimura J-I, et al. (2006) Molecular basis of clonal expansion of hematopoiesis in 2 patients with paroxysmal nocturnal hemoglobinuria (PNH). Blood 108: 4232-6.

13. Odero MD, Grand FH, Iqbal S, Ross F, Roman JP, et al. (2005) Disruption and aberrant expression of HMGA2 as a consequence of diverse chromosomal translocations in myeloid malignancies. Leukemia 19: 245-52.

14. Hristov AC, Cope L, Reyes MD, Singh M, Iacobuzio-Donahue C, et al. (2009) HMGA2 protein expression correlates with lymph node metastasis and increased tumor grade in pancreatic ductal adenocarcinoma. Mod Pathol 22: 43-9.

15. Di Cello F, Hillion J, Hristov A, Wood LJ, Mukherjee M, et al.(2008) HMGA2 participates in transformation in human lung cancer. Mol Cancer Res 6:743-50.

16. Nyquist KB, Panagopoulos I, Thorsen J, Roberto R, Wik HS, et al. (2012) t(12;13)(q14;q31) leading to HMGA2 upregulation in acute myeloid leukaemia. Br J Haematol 157: 769-71.

17. Murakami Y, Inoue N, Shichishima T, Ohta R, Noji H, et al. (2012) Deregulated expression of HMGA2 is implicated in clonal expansion of PIGA deficient cells in paroxysmal nocturnal haemoglobinuria. Br J Haematol 156: 383-7.

18. Kelly RJ, Tooze RM, Doody GM, Richardst SJ, Hillmen P (2008) The investigation of HMGA2 dysregulation and promoter mutations in PIG-M in the molecular pathogenesis of paroxysmal nocturnal haemoglobinuria (PNH). Brit J Haematol 141:110.

19. Shen W, Clemente MJ, Hosono N, Yoshida K, Przychodzen B, et al. (2014) Deep sequencing reveals stepwise mutation acquisition in paroxysmal nocturnal hemoglobinuria. J Clin Invest 124: 4529-38.

20. Nishimura J, Inoue N, Wada H, Ueda E, Pramoonjago P, et al. (1997) A patient with paroxysmal nocturnal hemoglobinuria bearing four independent PIG-A mutant clones. Blood 89: 3470-6.

21. Sellmann L, Scholtysik R, Kreuz M, Cyrull S, Tiacci E, et al. (2010) Gene dosage effects in chronic lymphocytic leukemia. Cancer Genet Cytogenet 203: 149-60.

22. Rauch A, Ruschendorf F, Huang J, Trautmann U, Becker C, et al. (2004) Molecular karyotyping using an SNP array for genomewide genotyping. J Med Genet 41: 916-22.

23. Zhao X, Li C, Paez JG, Chin K, Janne PA, et al. (2004) An integrated view of copy number and allelic alterations in the cancer genome using single nucleotide polymorphism arrays. Cancer Res 64: 3060-71.

24. Sutherland DR, Kuek N, Davidson J, Barth D, Chang H, et al. (2007) Diagnosing PNH with FLAER and multiparameter flow cytometry. Cytometry B Clin Cytom 72: 167-77. 
25. Martin-Subero JI, Harder L, Gesk S, Schlegelberger B, Grote W, et al. (2002) Interphase FISH assays for the detection of translocations with breakpoints in immunoglobulin light chain loci. Int J Cancer 98: 470-4.

26. Nückel H, Huttmann A, Klein-Hitpass L, Schroers R, Fuhrer A, et al. (2006) Lipoprotein lipase expression is a novel prognostic factor in B-cell chronic lymphocytic leukemia. Leuk Lymphoma 47: 1053-61.

27. Araten DJ, Swirsky D, Karadimitris A, Notaro R, Nafa K, et al. (2001) Cytogenetic and morphological abnormalities in paroxysmal nocturnal haemoglobinuria. Br J Haematol 115: 360-8.

28. Rosse WF (1997) Paroxysmal nocturnal hemoglobinuria as a molecular disease. Medicine (Baltimore) 76: 63-93.

29. Young NS, Abkowitz JL, Luzzatto L (2000) New Insights into the Pathophysiology of Acquired Cytopenias. Hematology Am Soc Hematol Educ Program: 18-38. 30. Bessler M, Mason P, Hillmen P, Luzzatto L (1994) Somatic mutations and cellular selection in paroxysmal nocturnal haemoglobinuria. Lancet 343: 951-3.

31. Nafa K, Bessler M, Castro-Malaspina H, Jhanwar S, Luzzatto L (1998) The spectrum of somatic mutations in the PIG-A gene in paroxysmal nocturnal hemoglobinuria includes large deletions and small duplications. Blood Cells Mol Dis 24: 370-84.

32. Mitelman F, Johansson B, Mertens F (2012) Mitelman Database of Chromosome Aberrations and Gene Fusions in Cancer, USA.

33. Longo L, Bessler M, Beris P, Swirsky D, Luzzatto L (1994) Myelodysplasia in a patient with pre-existing paroxysmal nocturnal haemoglobinuria: a clonal disease originating from within a clonal disease. Br J Haematol 87: 401-3.

34. Iwanaga M, Furukawa K, Amenomori T, Mori H, Nakamura H, et al. (1998) Paroxysmal nocturnal haemoglobinuria clones in patients with myelodysplastic syndromes. Br J Haematol 102: 465-74.

35. Dingli D, Luzzatto L, Pacheco JM (2008) Neutral evolution in paroxysmal nocturnal hemoglobinuria. Proc Natl Acad Sci U S A 105: 18496-500.

36. Mayr C, Hemann MT, Bartel DP (2007) Disrupting the pairing between let-7 and Hmga2 enhances oncogenic transformation. Science 315: 1576-9.

37. Ikeda K, Mason PJ, Bessler M (2011) 3'UTR-truncated Hmga2 cDNA causes MPN-like hematopoiesis by conferring a clonal growth advantage at the level of HSC in mice. Blood 117: 5860-9.

38. Bessler M, Hiken J (2008) The pathophysiology of disease in patients with paroxysmal nocturnal hemoglobinuria. Hematology Am Soc Hematol Educ Program: 10.1182/asheducation-2008.

39. Parker CJ (2009) Bone marrow failure syndromes: paroxysmal nocturnal hemoglobinuria. Hematol Oncol Clin North Am 23: $333-46$.

40. Gargiulo L, Papaioannou M, Sica M, Talini G, Chaidos A, et al. (2013) Glycosylphosphatidylinositol-specific, CD1d-restricted T cells in paroxysmal nocturnal hemoglobinuria. Blood 121: 2753-61.

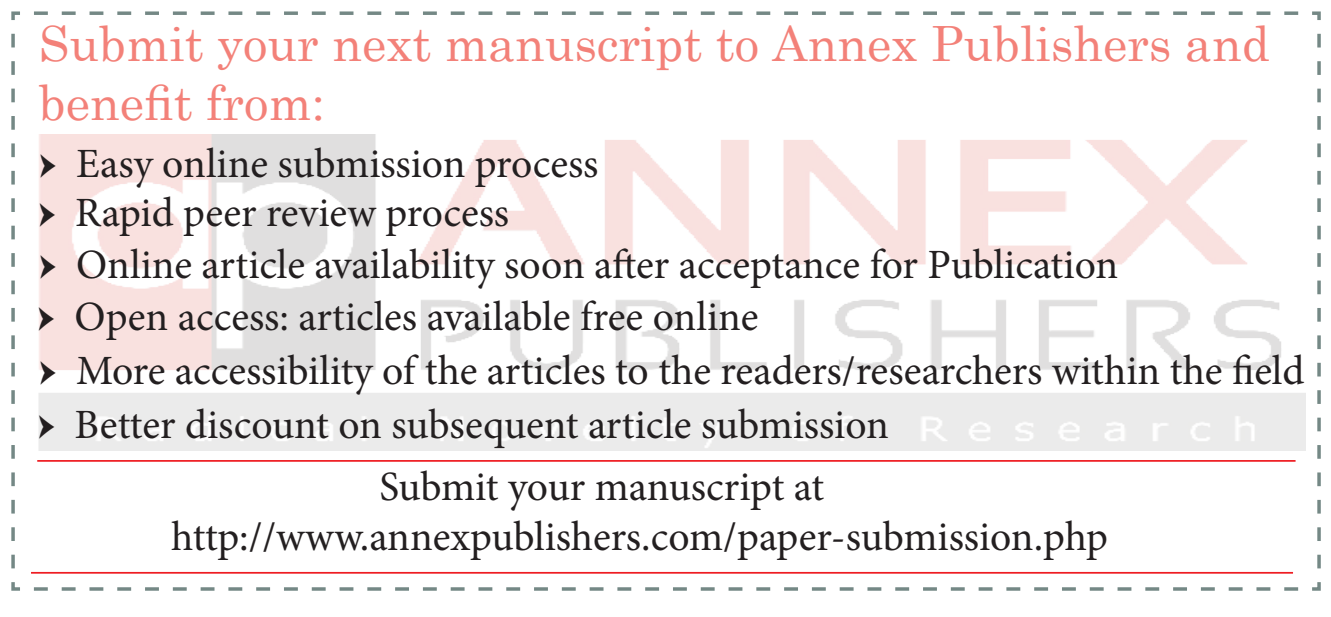

